\title{
Zur Sorption und Diffusion von Gasen in Poly-Äthylenglykol-Terephthalsäure-Ester
}

\author{
Von H.-Chr. Draisbach, D. Jeschke und H. A.Stuart \\ Aus dem Laboratorium für Physik der Hochpolymeren am Institut für physikalische Chemie \\ der Universität Mainz \\ (Z. Naturforschg. 17 a, 447-451 [1962]; eingegangen am 28. März 1962)
}

\begin{abstract}
Zur Ergänzung von früheren Bestimmungen der Löslichkeit $S$ und des Diffusionskoeffizienten $D$ von Gasen in Hochpolymeren nach der Barrerschen Permeationsmethode werden die beiden Größen durch direkte Sorptionsexperimente ermittelt. Die Messungen werden an amorphem und teilkristallinem Terylen durchgeführt; für $\mathrm{H}_{2}$ wird die Temperaturabhängigkeit von $S$ und $D$ beiderseits der Einfriertemperatur verfolgt.

Aus den Ergebnissen läßt sich schließen, daß die Art der Erniedrigung der beiden Größen infolge Kristallisation nicht im Zweiphasenmodell erklärt werden kann, und ferner, daß der Gastransport auch unterhalb der Einfriertemperatur durch Platzwechseldiffusion vonstatten geht. Aus den Mes. sungen der Temperaturabhängigkeit der beiden Größen lassen sich unter gewissen Voraussetzungen Lösungsenthalpien und Aktivierungsenergien berechnen.
\end{abstract}

Vor kurzem wurde hier über Untersuchungen des Durchganges von permanenten Gasen durch amorphe und teilkristalline Hochpolymere berichtet ${ }^{1}$. Die interessierenden Größen bei einer solchen Permeation von Gasen sind, entsprechend der Sorption und der Diffusion, aus denen sich der Permeationsvorgang bei reinen Platzwechselprozessen zusammensetzt, die Löslichkeit $S$, der Diffusionskoeffizient $D$ und der Permeationskoeffizient $P$. Sie wurden in der erwähnten Arbeit mittels der ,time-lag-Methode“ nach Barrer $^{2}$ bestimmt, wonach $P$ und $D$ aus der Messung des zeitlichen Gasdurchganges durch die Folie ermittelt werden, während $S$ sich aus der Beziehung $D S=P$ errechnet. Zur Kontrolle und zur Klärung einiger Fragen schien es jedoch interessant, die Sorp: tion und die Diffusion am gleichen Material auch getrennt zu untersuchen, d. h. die Löslichkeit in einem Absorptionsexperiment direkt zu messen und den Diffusionskoeffizient aus der Kinetik des Absorptionsvorganges nach DünNwald und WAGNer ${ }^{3}$ zu bestimmen. Über derartige Untersuchungen soll hier berichtet werden.

\section{Experimentelles}

Das Meßprinzip besteht darin, die zu untersuchende entgaste Polymerprobe, die als Folie vorliegen muß, mit dem betreffenden Gas bei bestimmter

1 D. Jeschie u. H. A. Stuart, Z. Naturforschg. 16 a, 37 [1961]; im folgenden mit AI beze:chnet.

2 R. M. Barrer, Diffusion in and through Solids, University Press, Cambridge 1951.
Temperatur und bestimmtem Gasdruck bis zur Sättigung in Kontakt zu bringen, sodann die Gasphase mit Quecksilber zu verdrängen und schließlich das in der Folie gelöste Gas in einem evakuierten Raum bekannten Volumens desorbieren zu lassen. Die Desorption äußert sich in diesem Desorptionsvolumen in einem Druckanstieg, der gegen einen Enddruck geht. Durch zeitliche Messung des Druckanstieges läßt sich der Desorptionsvorgang verfolgen, welcher zur Bestimmung des Diffusionskoeffizienten ausgewertet wird, während der Enddruck ein Maß für die abgegebene bzw. aufgenommene Gasmenge und damit für die Gaslöslichkeit $S$ ist. Die beiden Meß. größen werden in folgenden Einheiten angegeben: Diffusionskoeffizient $D$ in $\mathrm{cm}^{2} / \mathrm{sec}$, Löslichkeit $S$ als diejenige Gasmenge in $\mathrm{cm}^{3}$, reduziert auf Normalbedingungen, welche von $1 \mathrm{~cm}^{3}$ Polymermaterial bei einem Begasungsdruck von 1 atm bei $0{ }^{\circ} \mathrm{C}$ aufgenommen wird (Bunsenscher Absorptionskoeffizient); ihre Einheit ist also $\mathrm{Ncm}^{3} /\left(\mathrm{cm}^{3} \cdot \mathrm{atm}\right)$.

Die Apparatur, die für diese Messung erstellt wurde, ist eine Hochvakuumapparatur aus Glas und ähnelt weitgehend einer von Meares ${ }^{4}$ beschriebenen. Mittelpunkt ist die Meßzelle (Abb. 1) mit der Folie, die zu einem Hohlzylinder gebogen ist, und das McLeodManometer als Druckanzeigegerät. Die Meßzelle ist von einem Temperiermantel umgeben, so $\mathrm{da} \beta$ die Sorption bei verschiedenen Temperaturen von $20^{\circ} \mathrm{C}$ bis $100{ }^{\circ} \mathrm{C}$ ermöglicht wird. Zur Messung der Folientemperatur

3 H. Dünnwald u. C. Wagner, Z. Phys. Chem. B 24, 53 [1934]. 4 P. Meares, Trans. Faraday Soc. 54, 40 [1958]. 
ragt ein eingeschmolzenes Thermoelement in den Hohlzylinder hinein. Gasvorratskolben und das Pumpensystem vervollständigen die Anlage.

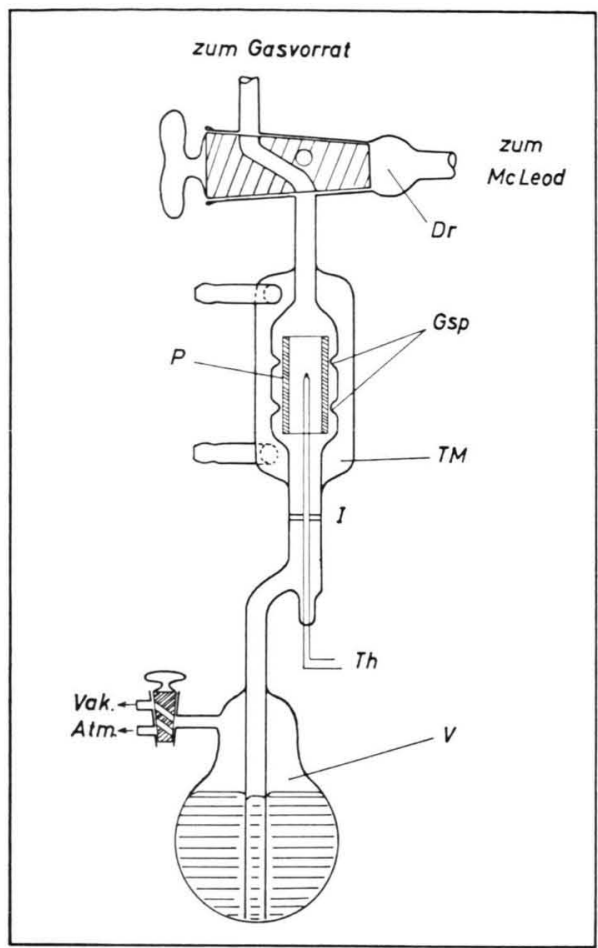

Abb. 1. Schema der Meßzelle. P Probe, TM Temperiermantel, Th Thermoelement, V Quecksilber-Vorratsgefäß, Dr Dreiwegehahn.

Die Begasungsdrucke variierten zwischen 50 und 500 Torr; innerhalb dieses Druckbereiches wurde das Henrysche Gesetz geprüft und für gültig gefunden, so daß es zur Bestimmung der absorbierten Gasmenge gleichgültig ist, bei welchem Begasungsdruck man innerhalb des geprüften Druckbereiches die Sättigung durchführt; zur Bestimmung von $S$ nach Definition muß dann noch auf den Begasungsdruck von 1 atm umgerechnet werden. Daß man die desorbierte Gasmenge der aufgenommenen gleichsetzen kann, folgt aus dem geringen Enddruck von $10^{-2}$ Torr im Desorptionsvolumen; daraus läßt sich die in der Probe zurückbleibende Gasmenge $\mathrm{zu} 10^{-3} \%$ der aufgenommenen abschätzen.

Zur Bestimmung des Diffusionskoeffizienten $D$ betrachtet man den Hohlzylinder näherungsweise als eine unendlich ausgedehnte Platte. Ist $D$ konzentrationsunabhängig und erfolgen Absorption und Desorption bei gleichem End- bzw. Anfangsdruck, so werden Absorption und Desorption identisch, und für die Abhängig-

5 W. Jost, Diffusion, Verlag Steinkopf, Darmstadt 1957, S. $55 \mathrm{ff}$.

6 Wir danken Herrn Dr. O. Herrmann von der K a ll e A G vielmals für die Überlassung des Materials.

7 Unter dem Kristallisationsgrad $\alpha^{\prime}$ verstehen wir den effek- keit der Konzentration von der Zeit gilt für beide Vorgänge folgende Gleichung ${ }^{5}$ :

$\frac{c_{\mathrm{e}}-c}{c_{\mathrm{e}}-c_{\mathrm{a}}}=\frac{8}{\pi^{2}} \sum_{n=0}^{\infty} \frac{1}{(2 n+1)^{2}} \exp \left\{-\left[(2 n+1) \frac{\pi}{d}\right]^{2} D t\right\}$

$\left(c_{\mathrm{e}}, c_{\mathrm{a}}\right.$ End- bzw. Anfangskonzentration in der Probe, $c$ mittlere Konzentration zur Zeit $t, d$ Dicke der Platte). Diese Reihe konvergiert so schnell, daß für genügend lange Zeiten nur das erste Glied berücksichtigt zu werden braucht. Durch Auftragen der Logarithmen des Bruches $\left(c_{e}-\bar{c}\right) /\left(c_{e}-c_{\mathrm{a}}\right)$ (der sich aus Meßwerten zusammensetzt) gegen die Zeit $t$ ergibt sich daher eine Gerade, deren Steigung dem Diffusionskoeffizienten proportional ist. Die Konzentrationsunabhängigkeit des Diffusionskoeffizienten wurde nachgeprüft, indem er bei einer bestimmten Temperatur für verschiedene Begasungsdrucke ermittelt wurde; er erwies sich dabei als konstant. Da außerdem zu Beginn der Absorption die Gasdrucke in der Probe gleich Null, im Außenraum gleich dem Begasungsdruck sind, während zu Beginn der Desorption die gleichen Drucke, aber vertauscht, herrschen, sind die Voraussetzungen zur Anwendung der Gl. (1) erfüllt.

Als Substanz wurde industriell hergestellter, praktisch amorpher Poly-Äthylenglykol-TerephthalsäureEster verwendet (Terylen), und zwar sog. „Hostaphan“. Vorfolie der Dicke $1 \mathrm{~mm}$ von der $\mathrm{Fa}$. $\mathrm{Ka}$ a l e A G ${ }^{6}$. Proben verschiedenen Kristallisationsgrades wurden erhalten, indem eine in der Meßzelle befindliche Folie durch Erhitzen mittels des Temperiermantels zum Kristallisieren gebracht wurde; nach Beendigung der Messungen wurde die Folie aus der Apparatur genommen und der Kristallisationsgrad $\alpha^{\prime}$ durch Messung der Dichte mittels der Schwebemethode bestimmt ${ }^{7}$. Auf diese Weise wurden alle Messungen mit $\alpha^{\prime}=30,6$ bzw. $43,0 \%$ durchgeführt.

Die verwendeten Gase waren $\mathrm{H}_{2}$ und $\mathrm{N}_{2}$ in technischer Reinheit; eventuelle Verunreinigungen wurden mit flüssiger Luft ausgefroren.

\section{Meßergebnisse und Diskussion}

\section{a) Löslichkeit}

Die Temperaturabhängigkeit der Löslichkeit für $\mathrm{H}_{2}$ in amorphem und teilkristallinem Terylen in einem Temperaturbereich beiderseits der Einfriertemperatur $\left(68{ }^{\circ} \mathrm{C}\right.$ für amorphes Terylen) zeigen die Abb. 2 und 3. Man erkennt eine Erniedrigung der Löslichkeit mit fortschreitender Kristallisation, außerdem zeigen die Kurven für den amorphen $\mathrm{Zu}$ stand ein ausgeprägtes Minimum in der Nähe der

tiven, d. h. den im Experiment wirksamen kristallinen Volumenanteil in Prozent, wie er in einem idealisierten Zweiphasensystem vorhanden wäre; er ist durch die Gleichung $\left(\varrho-\varrho_{\mathrm{a}}\right) /\left(\varrho_{\mathrm{kr}}-\varrho_{\mathrm{a}}\right)$ definiert $\left(\varrho\right.$ Dichte der Probe, $\varrho_{\mathrm{a}}$ bzw @ kr Dichte des rein amorphen bzw. rein kristallinen Stoffes). 


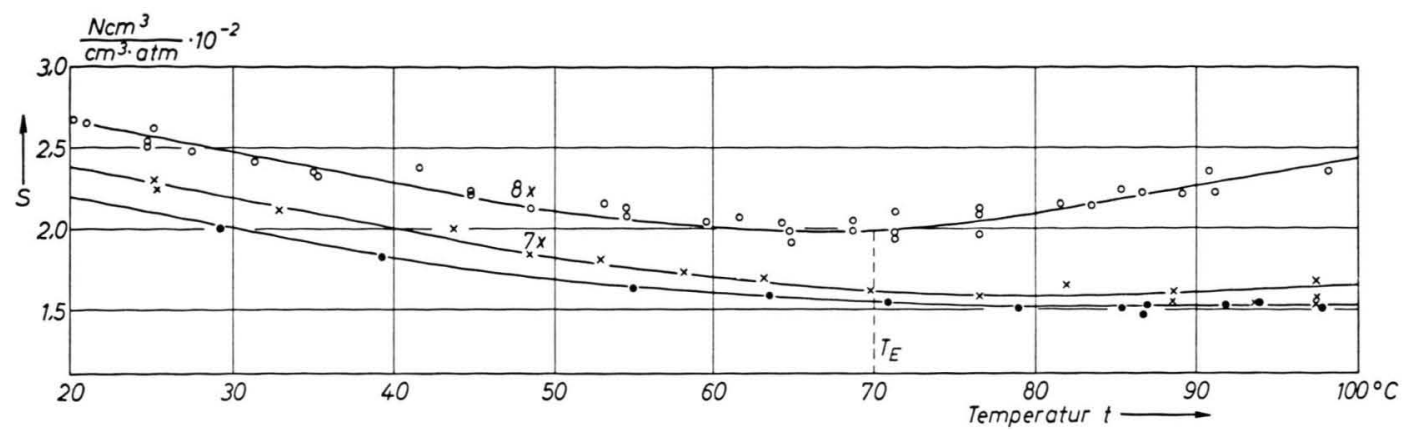

Abb. 2. Temperaturabhängigkeit der Löslichkeit von $\mathrm{H}_{2}$ in amorphem und teilkristallinem Terylen. $T_{\mathrm{E}}$ Einfriertemperatur. $\circ \circ \mathrm{H}_{2} /$ Terylen amorph, $\times \times \mathrm{H}_{2} /$ Terylen krist. $\left(\alpha_{\mathrm{I}^{\prime}}=30,6 \%\right)$, $\bullet \mathrm{H}_{2} /$ Terylen krist. $\left(\alpha_{\mathrm{II}}{ }^{\prime}=43,0 \%\right)$.

Einfriertemperatur, das sich mit fortschreitender Kristallisation verflacht. Messungen mit $\mathrm{N}_{2}$ an den gleichen Proben wurden nur bei der Temperatur von $65{ }^{\circ} \mathrm{C}$ ausgeführt, vgl. Tab. 1 .

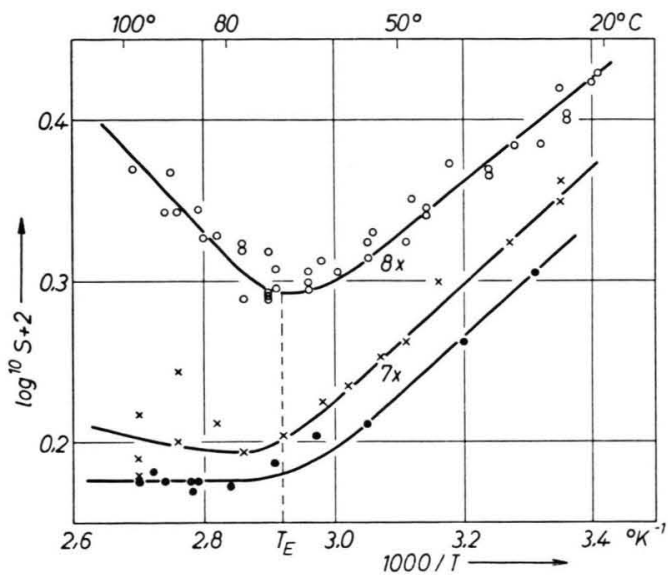

Abb. 3. Auftragung der Werte von Abb. 2 als $\log S$ gegen die reziproke absolute Temperatur. $T_{\mathrm{E}}$ Einfriertemperatur. Bezeichnung der Meßpunkte wie in Abb. 2.

Soweit die Löslichkeit $S(T)$ die Gleichgewichtskonstante für ein wirkliches Lösungsgleichgewicht zwischen der Gasphase und der Lösungsphase darstellt, kann man aus Messungen der Temperaturabhängigkeit mit Hilfe der Gleichung der Reaktionsisobaren

$$
\frac{\mathrm{d}}{\mathrm{d} T} \ln S(T)=\frac{\Delta H}{R T^{2}}
$$

die Lösungsenthalpie $\Delta H$ bestimmen, vgl. die Zahlenwerte der Tab. 2. Oberhalb der Einfriertemperatur verläuft der Lösungsvorgang endotherm (positive Enthalpiewerte). Der unterhalb der Einfriertemperatur beobachtete Wiederanstieg der Löslichkeit mit abnehmender Temperatur, der auch von

\begin{tabular}{|c|c|c|}
\hline Terylenprobe & $S \cdot 10^{2}$ & $D \cdot 10^{8}$ \\
\hline amorph & 2,38 & 1,5 \\
$\alpha^{\prime}=30,6 \%$ & 2,31 & 0,9 \\
$\alpha^{\prime}=43,0 \%$ & 2,26 & 0,6 \\
\hline
\end{tabular}

Tab. 1. $S$ und $D$ von $\mathrm{N}_{2}$ in amorphem und teilkristallinem Terylen bei $65^{\circ} \mathrm{C}$ (Dimensionen siehe Abschnitt II).

\begin{tabular}{|c|c|c|c|c|}
\hline \multirow{3}{*}{$\begin{array}{l}\text { Terylen- } \\
\text { probe }\end{array}$} & \multicolumn{2}{|c|}{$\Delta H$ in $\mathrm{cal} / \mathrm{gmol}$} & \multicolumn{2}{|c|}{$E_{\mathrm{D}}$ in $\mathrm{cal} / \mathrm{gmol}$} \\
\hline & unterhalb & oberhalb & unterhalb & oberhalb \\
\hline & \multicolumn{2}{|c|}{$\begin{array}{l}\text { der Einfrier- } \\
\text { temperatur }\end{array}$} & \multicolumn{2}{|c|}{$\begin{array}{l}\text { der Einfrier- } \\
\text { temperatur }\end{array}$} \\
\hline $\begin{array}{c}\text { amorph } \\
\alpha^{\prime}=30,6 \% \\
\alpha^{\prime}=43,0 \%\end{array}$ & $\begin{array}{l}(-1700) \\
(-1900) \\
(-2000)\end{array}$ & $\begin{array}{c}+1900 \\
- \\
-\end{array}$ & $\begin{array}{l}6900 \\
6400 \\
5400\end{array}$ & $\begin{array}{l}9600 \\
9200 \\
8700\end{array}$ \\
\hline
\end{tabular}

Tab. 2. Lösungsenthalpien $\Delta H$ und Aktivierungsenergien $E_{\mathrm{D}}$ von $\mathrm{H}_{2}$ in amorphem und teilkristallinem Terylen.

Meares ${ }^{4,8}$, und zwar an amorphem Polyvinylchlorid für verschiedene Inertgase gefunden wurde, hängt offenbar mit dem Einfrieren der mikrobrownschen Bewegung unterhalb der Einfriertemperatur zusammen. Diese Erscheinung führt bekanntlich zu einer lockeren Packung der Molekülsegmente, d. h. $\mathrm{zu}$ Packungslücken oder Spalten von molekularen Abmessungen („Löcherbildung“). Die Annahme liegt nahe, daß solche Lücken mit Gasmolekülen aufgefüllt werden, und daß vielleicht zusätzlich eine Adsorption an inneren Oberflächen stattfindet.

Da die Dichte im eingefrorenen Zustand mit abnehmender Temperatur immer mehr von ihrem Gleichgewichtswert abweicht, die „Löcherbildung“ also ansteigt, wird immer mehr Gas zusätzlich auch dann aufgenommen, wenn mit dieser zusätzlichen

8 P. Meares, J. Amer. Chem. Soc. 76, 3415 [1954]. 


\begin{tabular}{|c|c|c|}
\hline & $\begin{array}{l}\text { Ergebnisse aus } \\
\text { Desorptionsmessungen } \\
\text { (an } 1 \mathrm{~mm} \text { dicker Folie) }\end{array}$ & $\begin{array}{c}\text { Ergebnisse aus } \\
\text { Permeationsmessungen } \\
\text { (an } 120 \mu \text { dicker Folie }\end{array}$ \\
\hline $\begin{array}{l}S \text { in } \mathrm{Nem}^{3} / \mathrm{cm}^{3} \text { atm } \\
\text { für den amorphen Zustand }\end{array}$ & $2,7 \cdot 10^{-2}$ & $7,2 \cdot 10^{-2}$ \\
\hline $\begin{array}{l}D \text { in } \mathrm{cm}^{2} / \mathrm{sec} \\
\text { für den amorphen Zustand }\end{array}$ & $4,0 \cdot 10^{-7}$ & $3,9 \cdot 10^{-7}$ \\
\hline $\begin{array}{l}\Delta H \text { in cal/gmol } \\
\text { unterhalb der Einfriertemperatur } \\
\text { amorph } \\
\text { teilkrist. } 1 \\
\text { teilkrist. } 2\end{array}$ & $\begin{array}{ll}(-1700) & \left(\alpha^{\prime}=0\right) \\
(-1900) & \left(\alpha^{\prime}=30,6 \%\right) \\
(-2000) & \left(\alpha^{\prime}=43,0 \%\right)\end{array}$ & $\begin{array}{l}(-1900)\left(\alpha^{\prime}=0\right) \\
-\overline{1800)}\left(\alpha^{\prime}=40 \%\right)\end{array}$ \\
\hline $\begin{array}{l}E_{\mathrm{D}} \text { in cal/gmol } \\
\text { unterhalb der Einfriertemperatur } \\
\text { amorph } \\
\text { teilkrist. } 1 \\
\text { teilkrist. } 2\end{array}$ & $\begin{array}{ll}6900 & \left(\alpha^{\prime}=0\right) \\
6400 & \left(\alpha^{\prime}=30,6 \%\right) \\
5400 & \left(\alpha^{\prime}=43,0 \%\right)\end{array}$ & $\begin{array}{ll}7300 & \left(\alpha^{\prime}=0\right) \\
7000 & \left(\alpha^{\prime}=32 \%\right) \\
6900 & \left(\alpha^{\prime}=40 \%\right)\end{array}$ \\
\hline $\begin{array}{l}E_{\mathrm{D}} \text { in cal/gmol } \\
\text { für den amorphen Zustand }\end{array}$ & & $\begin{array}{l}\text { (Material: } \\
1 \text { mm dicke Folie) }\end{array}$ \\
\hline $\left.\begin{array}{l}\text { unterhalb } \\
\text { oberhalb }\end{array}\right\}$ der Einfriertemperatur & $\begin{array}{l}6900 \\
9600\end{array}$ & $\begin{array}{l}4900 \\
9600\end{array}$ \\
\hline
\end{tabular}

Tab. 3. Vergleich der aus Permeations- ${ }^{1}$ und Desorptionsmessungen gewonnenen Werte der Löslichkeit, des Diffusionskoeffizienten, der Lösungsenthalpie und der Aktivierungsenergie an „Hostaphan“-Vorfolie.

Löslichkeit überhaupt kein Wärmeeffekt verbunden ist. Im Hinblick auf diesen mit der Strukturänderung der Probe unterhalb der Einfriertemperatur verbundenen Auffülleffekt kann man daher aus dem Kurvenverlauf auch keine Lösungsenthalpie nach einer nur für ein wirkliches Gleichgewicht gültigen Beziehung berechnen. Die aus der Sättigung berechneten Werte haben daher ohne zusätzliche Informationen keinen physikalischen Sinn und sind deshalb in Tab. 2 und 3 eingeklammert.

Die Abnahme der Löslichkeit mit zunehmender Kristallisation läßt sich nicht an Hand eines einfachen Zweiphasenmodells erklären, bei dem man voraussetzt, daß Sorption und Diffusion nur in der amorphen Phase erfolgen. Nach diesem Modell müßte die relative Löslichkeit $S_{\mathrm{r}}$ (d. i. die Löslichkeit $S_{\mathrm{kr}}$ im teilkristallinen Material bezogen auf die Löslichkeit $S_{\mathrm{a}}$ im amorphen) linear mit dem amorphen Anteil $1-\alpha^{\prime}$ abnehmen und außerdem von der Gasart und der Temperatur unabhängig sein. Wie die Abb. 4 zeigt, ist das nicht der Fall. Eine mögliche Erklärung ist, daß in den schlecht geordneten kristallinen Bereichen sowohl Sorption wie Diffusion möglich sind; über weitere Modellvorstellungen vgl. AI.

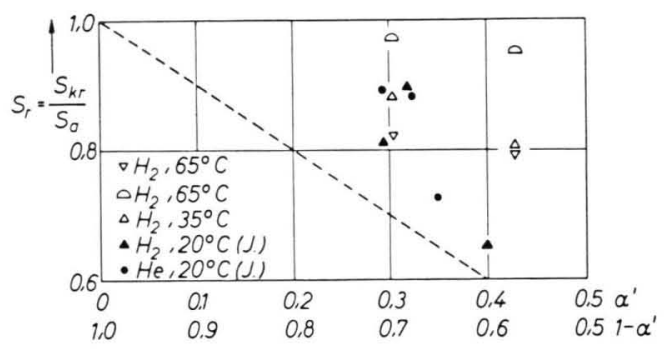

Abb. 4. Erniedrigung der Löslichkeit mit fortschreitender Kristallisation. Messungen von J EschKe ( $\mathrm{vgl}$. AI) sind mit J. bezeichnet. $\bigcirc \mathrm{N}_{2}, 65{ }^{\circ} \mathrm{C}$ (nicht $\mathrm{H}_{2}$ ).

\section{b) Diffusionskoeffizient}

Auch beim Diffusionskoeffizienten finden wir eine Abnahme mit fortschreitender Kristallisation. Im einfachen $Z$ weiphasenmodell müßte der relative Diffusionskoeffizient nur eine Funktion des Verhältnisses kristallin-amorph und insbesondere unabhängig von der Gasart sein, was durch unsere Messungen nicht bestätigt wird, siehe Abb. 5. Die Abhängigkeit des Diffusionskoeffizienten vom Kristallisationsgrad ist allerdings eine andere und viel kompliziertere als diejenige der Löslichkeit, vgl. AI.

Die Abb. 6 zeigt die Temperaturabhängigkeit des Diffusionskoeffizienten von $\mathrm{H}_{2}$ in amorphem und 


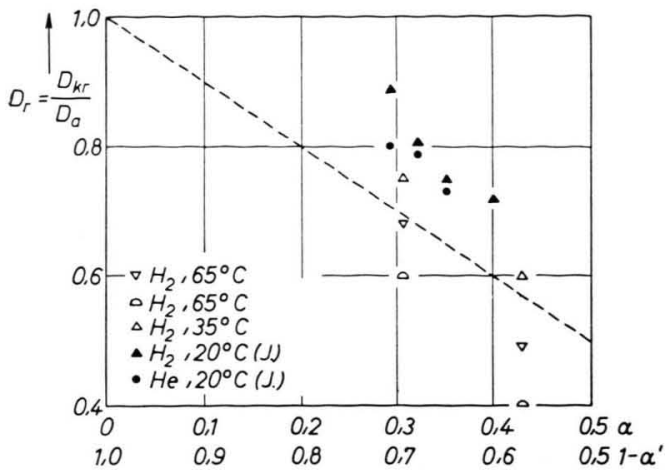

Abb. 5. Erniedrigung des Diffusionskoeffizienten mit fortschreitender Kristallisation. Messungen von JеschKe (vgl. AI) sind mit J. bezeichnet. $\triangle \mathrm{N}_{2}, 65{ }^{\circ} \mathrm{C}$ (nicht $\mathrm{H}_{2}$ ).

teilkristallinem Material, aufgetragen als $\log D$ gegen die reziproke absolute Temperatur. Man sieht daraus: Der Erhöhung der Löslichkeit unterhalb

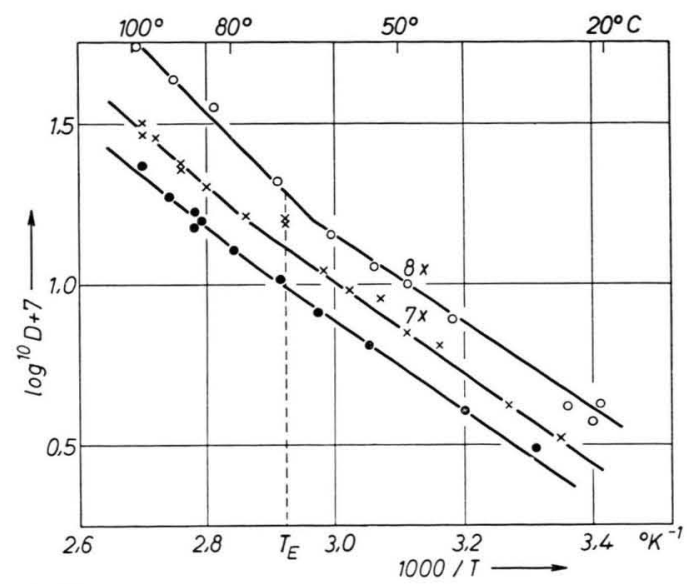

Abb. 6. Temperaturabhängigkeit des Diffusionskoeffizienten von $\mathrm{H}_{2}$ in amorphem und teilkristallinem Terylen, aufgetragen als $\log D$ gegen die reziproke absolute Temperatur. $T_{\mathrm{E}}$ Einfriertemperatur. Bezeichnung der Meßpunkte wie in Abb. 2. Es ist ein Meßpunkt $\bigcirc$ mit der Ordinate 0,75 und der Abszisse 1000/T $=3,25$ nachzutragen. der Einfriertemperatur infolge „Löcherbildung“ entspricht eine Zunahme des Diffusionskoeffizienten gegenüber dem Wert, wie er ohne Einschränkung der mikrobrownschen Bewegung zu erwarten wäre. Da man aus Abb. 6 sowohl unterhalb wie oberhalb der Einfriertemperatur Arrheniussche Geraden mit verschiedener Steigung erkennt, die sich in der Nähe der Einfriertemperatur schneiden, liegt auch unterhalb der Einfriertemperatur eine Platzwechseldiffusion vor; doch wird man dabei einen Platzwechselprozeß mit 2 verschiedenen Elementarschritten $\lambda$ der Diffusion im Sinne der Eyringschen Platzwechseltheorie annehmen müssen. Aus der Neigung der Geraden lassen sich gemäß einer Arrheniusschen Gleichung

$$
D=D_{0} \exp \left(-E_{\mathrm{D}} / R T\right)
$$

Aktivierungsenergien der Diffusion berechnen, welche allerdings wegen der verschiedenen Anteile, aus denen sich eine derart empirisch bestimmte Aktivierungsenergie zusammensetzt, besser als scheinbare (apparent) Aktivierungsenergien bezeichnet werden.

Schließlich stellen wir in der Tab. 3 die nach der Desorptions- und nach der Permeationsmethode bestimmten Meßwerte von $S$ und $D$ sowie die aus deren Temperaturabhängigkeit berechneten Lösungsenthalpien und Aktivierungsenergien zusammen. Die Übereinstimmung ist i. allg. befriedigend, zumal wenn man beachtet, daß die Foliendicke und damit der Vorordnungsgrad verschieden waren; die Permeationsmessungen wurden nämlich an "Hostaphan"-Vorfolien durchgeführt, deren Dicke $120 \mu$ betrug. Eine Ausnahme hiervon bilden lediglich die Werte der Aktivierungsenergien in den beiden letzten Zeilen der Tab. 3, welche aus Messungen an der $1 \mathrm{~mm}$ dicken Folie gewonnen wurden. 